\title{
POSICIONAMIENTO CULTURAL E IDENTIDAD CULTURAL COMO PRINCIPIOS DE UNA ENSEÑANZA BASADA EN CONTENIDOS EN LA CLASE DE INGLÉS COMO LENGUA EXTRANJERA
}

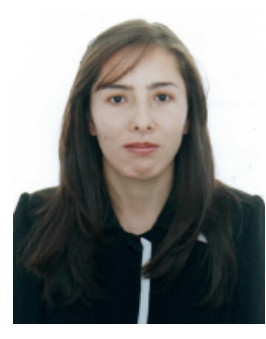

Fecha de recepción:26/02/2020
Fecha de revisión:22/03/2020
Fecha de aceptación:23/05/2020

\section{RESUMEN}

Este estudio muestra como el conocimiento cultura es representado por los estudiantes de noveno grado de un colegio público de Floresta (Boyacá-Colombia) al desarrollar un aprendizaje basado en contenidos durante las clases de inglés como lengua extranjera. La investigación es un estudio de caso que busca entender y describir la cultura de los participantes siguiendo el paradigma de la investigación cualitativa. Ochenta estudiantes son los beneficiarios del desarrollo de la intervención pedagógica, en el área de inglés, pero de ellos solo cinco estudiantes se constituyen en los participantes del estudio teniendo en cuenta un parámetro de regularidad durante la intervención pedagógica. Los datos se recogieron a través de diarios de los participantes y una entrevista en profundidad, esta última para analizar detalladamente los temas que surgían de los diarios. La intervención Pedagógica estuvo basada en el diseño de una unidad curricular sobre los artefactos culturales del municipio como mediadores para mejorar las habilidades de aprendizaje de una lengua extranjera. Como resultado del proceso de análisis de datos y la interpretación de las voces de los participantes surgieron dos categorías (la primera es Conocer y Entender mi Cultura y la segunda es Mi Herencia Cultural como Componente de mi Identidad) que dan cuenta de la pregunta de investigación. Los resultados evidencian un posicionamiento de los participantes frente al hecho de ser dueños de una cultura y la responsabilidad de preservar su memoria cultural.

Palabras clave: Lenguaje, Cultura, Conciencia Cultural, Instrucción basada en contenidos. 


\title{
CULTURAL POSITIONING AND CULTURAL IDENTITY AS PRINCIPLES OF A CONTENT-BASED EDUCATION IN THE CLASS OF ENGLISH AS A FOREIGN LANGUAGE
}

\begin{abstract}
This study shows how particular cultural knowledge is portrayed by Ninth grades at a public school in Floresta (Boyacá-Colombia) when developing a content-based instruction in EFL classes. The inquiry is a case study that aims to understand and describe participants' culture following the qualitative research paradigm. Eighty students are the participants in the pedagogical intervention, in the English subject matter, but only five of them are the participants and data informers of the study because of a regularity parameter during the pedagogical intervention. The data were collected through participants' journals and in-depth interview, the last one to analyze in-depth journals' emerging themes. The pedagogical intervention consisted in the design of a curricular unit addressing the local culture artifacts as mediators to improve foreign language-learning skills. As a result of the data analysis process and interpretation of the participants' voices two categories emerged (the first one, Knowing and understanding my culture and the second is My Cultural Heritage as Component of my Identity) that give account of the research question. The results show a participants' positioning in front of being owners of culture and the responsibility to keep their cultural memory.
\end{abstract}

Key words: Language, Culture, Cultural Identity, Content-based Learning

\section{INTRODUCCIÓN}

Este documento presenta un estudio realizado en una institución educativa pública del departamento de Boyacá-Colombia y explora la forma en que los estudiantes describen su conocimiento cultural, las reflexiones que emergen en torno a la identidad cultural y las implicaciones de estos posicionamientos para asumir un rol. Además, este estudio muestra el diseño de una unidad curricular, en un contexto donde el inglés se enseña como lengua extranjera, para usar los artefactos culturales locales como pretextos del aprendizaje de otra lengua.

La idea de esta investigación surge al identificar que las prácticas pedagógicas en el área de inglés estaban apartadas totalmente del contexto local y que esto hacia que el aprendizaje del inglés fuese descontextualizado, careciera de relevancia para los participantes y que encontraran, en algunos casos, el aprendizaje del inglés como una práctica alejada de sus realidades y posibilidades. Siguiendo los parámetros de una enseñanza sensible a la realidad de los aprendices y que cobre 
relevancia en sus vidas académicas y profesionales, el docente-investigador de este estudio comprende la urgente necesidad de involucrar características del contexto local al currículo y planeación de aula; más importante aún, se determina una imperiosa urgencia por involucrar a los estudiantes en prácticas de aprendizaje significativas que les permitan, además de aprender otra lengua, reflexionar sobre su realidad y la forma en que ellos son portadores de una cultura y/o constructores de la misma.

En la mayoría de las aulas de clase de aprendizaje de una lengua, la cultura ha tomado un papel trascendental dada la relevancia que han tenido las teorías de Sonia Nieto (2002) al proponer el lenguaje como un medio que provee las costumbres, normas, artefactos e información importante dentro del desarrollo social de una comunidad que es perpetuado, construido y resignificado de generación en generación.

Desde esta consideración, se traza como objetivo central de este trabajo la identificación y caracterización del conocimiento cultural e identidad cultural de los participantes desde las reflexiones que emergen fruto del desarrollo de una práctica pedagógica de aula pensada con una finalidad esencial: aprendizaje del ingles desde la reflexión sobre temas locales y su incidencia en la vida personal, familiar y social de los estudiantes. Por tanto, se hace indispensable el desarrollo de unas acciones previas que permitan alcanzar este propósito entre las que se cuenta el diseño de una unidad curricular, la implementación de la misma en las sesiones de clase y el desarrollo de diarios estudiantiles y entrevistas como instrumentos de recolección de información.

\section{FUNDAMENTOS TEÓRICOS}

Dada la intencionalidad dual de este estudio se hace imperioso entablar una discusión teórica en la que, primero, se comprendan los conceptos de lenguaje, cultura e identidad para la caracterización de la identidad de los participantes y, segundo, una aproximación a la enseñanza basada en contenidos que permita el desarrollo de la unidad curricular como sustento instruccional del presente estudio. Inicialmente, debe considerarse que los conceptos de lenguaje, cultura e identidad tienen una relación estrecha al ser parte fundamental de la realidad humana; de esta forma, en la discusión teórica se encontraran puntos comunes en los que los tres conceptos convergen.

\section{LENGUAJE}

Desde los inicios de la vida humana, el lenguaje ha sido el vehículo para nombrar el mundo que nos rodea, un medio para conocer el pensamiento de la humanidad. Es una realidad que éste se ha constituido en uno de los asuntos más estudiados por los lingüistas y que las teorías han evolucionado, así como el concepto de lenguaje ha cambiado. Para el propósito de este documento 
es crucial entender algunas conceptualizaciones que permitan luego dilucidar las implicaciones de la enseñanza del lenguaje para, entonces, aproximarnos a la relación entre lenguaje, cultura e identidad.

Inicialmente, el lenguaje fue conceptualizado como un sistema de elementos bien organizados que se ajustaban a construcciones gramaticales (Chomsky, 1965); más adelante, Halliday (1973) define el lenguaje como un discurso de significado potencial, que, en otras palabras, es un conjunto de significados logrados por el oyente-hablante inmerso en un contexto socio-cultural. Más recientemente, las teorías se orientan a un concepto de lenguaje relacionado con la "ideología" (Hodge, 1999; Thomson, 1990; Kroskrity, 2000) donde el "lenguaje es uno de los atributos más notables del hombre. Éste es una condición absoluta de casi toda nuestra vida social y es, también, el medio en el cual los pensamientos y comunicación más organizados tienen lugar” (Hodge.p.56)

Retomando el último concepto en el que se remarca la condición social del lenguaje y se resalta el hecho de que todas las relaciones humanas están permeadas por el lenguaje, se puede llegar a la afirmación que el lenguaje se constituye en un vehículo para entender, reflexionar, analizar y construir la realidad de las personas entre las que se encuentra la cultura y el lenguaje mismo. Kramsch (1998), citado por Moran (2001), usa la metáfora del lenguaje como vehículo para ilustrar que "los miembros de una cultura han creado el lenguaje para llevar a cabo sus prácticas culturales, para identificar y organizar todos los aspectos culturales de su entorno y para nombrar las perspectivas inmersas en todas las diversas comunidades que abarcan la cultura" (p.35).

En este contexto de educación bilingüe, es importante entender el lenguaje no solo como la capacidad humana de producir sonidos sino como la forma de representar o crear ideas sobre el mundo circundante debido a su naturaleza de fenómeno social y cultural (Hinkel, p.9); para reafirmar esta idea, Hymes (1996) considera que "el lenguaje juega un papel importante en la vida social de una comunidad" no solo para establecer las relaciones humanas sino también para construir identidades propias y colectivas.

En esta perspectiva, el lenguaje es no solo una construcción social sino también una ideología y filosofía; el lenguaje es un atributo personal que determina la mayoría, sino todos los comportamientos, pensamientos y actitudes humanas para ser convertidos en conocimiento o experiencias. En la misma línea de pensamiento, Hodge (1999) plantea que los seres humanos han usado el lenguaje como ideología para representar, sentir o creer acerca del mundo y acerca de las relaciones que las personas establecen a través del lenguaje; así mismo, Hinkel (1999) y Hymes (1996) proponen que el lenguaje es parte del proceso de socialización al ser conceptuado como fenómeno social y cultural. 
Debido a la importancia del lenguaje en la comprensión y formación de los sistemas culturales y sociales de los seres humanos llama la atención el hecho de que todas las prácticas educativas y sus actores de enseñanza (docentes) están llamados a fortalecer acciones pedagógicas donde el aprendizaje del lenguaje ofrezca oportunidades de empoderamiento en el desarrollo de habilidades de comunicación, cognición y afectividad sumadas a un conocimiento cultural foráneo que no deje de lado el local, por su puesto. ( Zuluaga et al, 2009; Álvarez \& Bonilla, 2009).

\section{CULTURA}

Gran número de teorías recientes consideran una perspectiva más amplia del término cultura de los que hace varios años se proponía; la cultura es conceptuada como el conocimiento de los elementos particulares que son portadores de significados importantes para una comunidad, y por ende, para sus miembros. Nieto (2002); partiendo de esta idea, y para los propósitos de este estudio, la cultura es entendida como un "emblema dinámico" que identifica a las personas como miembros de una comunidad o ideología particular. Hinkel (1999) conceptualiza a la cultura como "no estática", que evoluciona al tiempo que las personas dan curso a sus vidas e interpretan sus experiencias sociales y culturales de diversas formas. (p.77).

El término cultura lleva consigo un significado semántico en el que se consideran todas las actividades, elementos, creencias, actitudes, comportamientos, pensamientos, tradiciones y casi todos los comportamientos humanos como constitutivos de la realidad de las comunidades y sus miembros. Es importante recordar que gran parte de los elementos mencionados anteriormente son transmitidos, construidos o heredados pero que aun así no permanecen siendo los mismo con el paso del tiempo (Nieto, 2002). Así, la cultura da cuenta de los siguientes elementos:

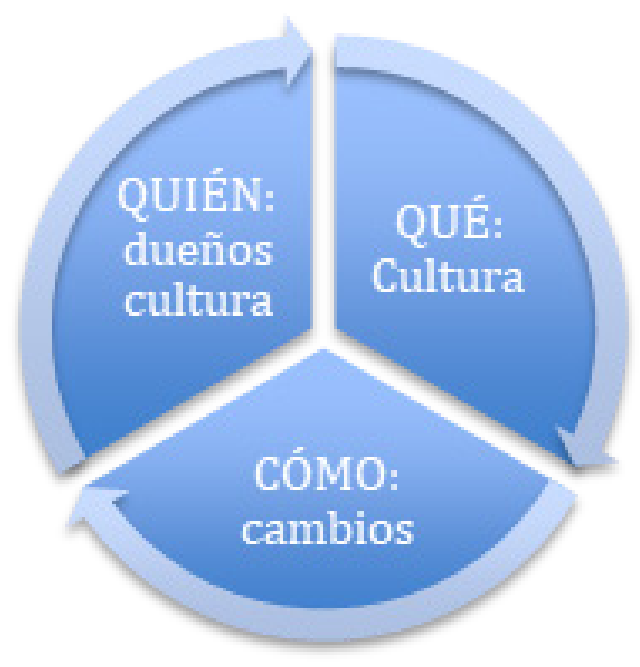

ORATORES ISSN Impreso: 2410-8928 ISSN Electrónico: L-2644-3988 Año 8. Número 12. Junio - Noviembre 2020 
Hinkel y Nieto declaran que la conceptualización de cultura va más allá de la construcción de una lista de rituales, creencias, tradiciones, festivales, aunque estos sean constitutivos de dicho concepto y resaltan la necesidad de abordar el concepto hacia una visión dinámica y cambiante compartida por una comunidad. Desde esta misma mirada, Nieto (2002) agrega que "la cultura es dinámica, activa, cambiante, siempre en transformación" (p.11). Bajo estos postulados, la información provista por los participantes muestra que ellos perpetúan algunas tradiciones pero que, al mismo tiempo, proponen unas conceptualizaciones propias sobre su cultura y su incidencia en sus vidas como miembros de una comunidad particular.

En este camino de comprensión teórica del término cultura es importante resaltar sus características para tener la visión amplia del término:
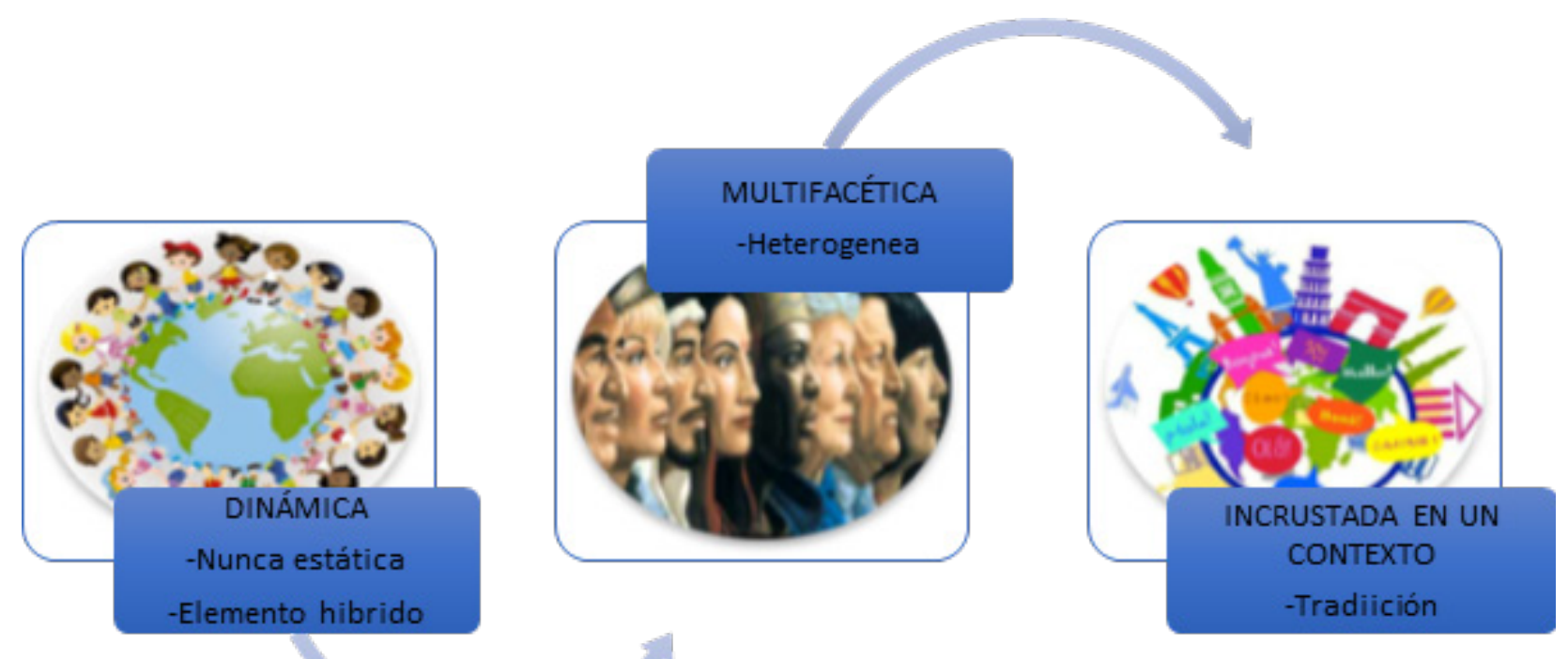

\section{IDENTIDAD}

La identidad ha sido asunto de las prácticas pedagógicas de enseñanza-aprendizaje de una lengua ya que es reconocido que los individuos expresan su identidad, así como su cultura, por medio del lenguaje. Cuando se hace uso del lenguaje, el ser humano no solo comunica sino que afirma y construye una posición propia frente a su relación con la comunidad, la cultura y su ser como sujeto social.

Desde la reflexión conceptual de lenguaje y cultura, se colige una condición indispensable en los dos: se constituyen en identidad del ser humano desde su esencia de constructos sociales. Desde aquí, reconocemos en la identidad "un conocimiento construido desde el ámbito sociocultural, un entendimiento que emerge de la relación de las personas con el contexto y la experiencia" (Norton, 2006). 
Norton (1997) define la identidad como "la manera en que las personas comprenden su relación con el mundo y cómo esa relación es construida a lo largo del tiempo y el espacio entendiendo sus posibilidades a futuro" (p.19). De esta manera, la identidad se manifiesta en todos los comportamientos, desde los físicos a los ideológicos, que un individuo desarrolla a lo largo de su existencia.

El concepto de identidad, tal como lo plantea Cakir (2006), es una "huella que guía el comportamiento de las personas en una comunidad y es engendrado en el escenario familiar". La identidad determina el comportamiento de los individuos, los hace más conscientes de las condiciones de estatus y roles; la identidad nos ayuda a reconocer que tan lejos podemos desarrollarnos como individuos y cuál es nuestra participación dentro de un grupo social. (p.79).

De manera similar a la cultura, la identidad esta en continua construcción por lo que se caracteriza por ser dinámica y estar en continuo cambio y construcción. Coherentemente con el lenguaje y la cultura, la identidad es un elemento móvil que responde a un contexto y escenarios particulares. Ya que los seres humanos no son iguales ni permanecen así en el tiempo, sus identidades también se están redefiniendo todo el tiempo.

Pavlenko (2004) resalta que la identidad construye y es construida por el lenguaje; a través del lenguaje, las personas afirman sus identidades y delimitan la manera en que se identifican dentro de un grupo social (p.54). De igual forma, Norton (1997) agrega,

“...cada vez que los hablantes de una lengua se comunican, no solo están intercambiando información con sus interlocutores; también están continuamente organizando y reorganizando un sentido de quiénes son y cómo se relacionan con un entorno social. En otras palabras, ellos se están involucrados en la construcción de su identidad a través de la negociación de sentidos y conceptos". (p.410).

Forés (2013) señala que "la identidad es un constructo dinámico, lleno de tensiones y transformaciones. En cada ser conviven múltiples identidades que configuran la IDENTIDAD en mayúsculas, aquello que «soy». La(s) identidad(es) se construyen dentro de un proceso de socialización, en los espacios de interacción mediante identificaciones y atribuciones". (p.10). En estas líneas teóricas se hace evidente la importancia de apuntar a prácticas de enseñanza-aprendizaje del inglés, o cualquier otro idioma, que promuevan una identidad cultural, mediante el uso de la riqueza contextual local, y, por ende, un escenario de sociedades heterogéneas sensibles donde cada individuo es valorado y valora la diferencia como escenarios constructivos. 


\section{METODOLOGÍA DEL ESTUDIO}

Este estudio se orientó bajo los principios de la investigación cualitativa "para estudiar los fenómenos en su escenario natural, buscando construir un sentido de o interpretar un fenómeno en términos de los significados que las personas le atribuyen a dicho evento" (Denzin, 2005, p.74). En un estudio cualitativo como este, tal como lo postula Burns (2000), el investigador permite que los participantes hablen con su "propia voz" y provean sus perspectivas del fenómeno de estudio; de esta forma, en este estudio se estableció como actividad central escuchar las reflexiones y pensamientos de los estudiantes frente a su aprendizaje del inglés y su construcción y/o reafirmación de una identidad y conocimiento cultural.

Yin (2003) ilumina el tipo de estudio de este proyecto: estudio de caso. Considerado como una indagación empírica que investiga el fenómeno dentro de un contexto real, el principal propósito del estudio de caso es obtener una comprensión de la reflexión de los estudiantes que permita obtener un aprendizaje frente a la práctica pedagógica y la identidad cultural de los participantes. De esta forma, los datos recolectados se obtuvieron desde el uso de un método mixto de recolección de información (diarios de los estudiantes y entrevistas, una general y una focalizada por participante) que fueron triangulados para el establecimiento de categorías de análisis, que se muestran en los resultados.

\section{"MI CULTURA FLORESTANA": PROPUESTA METODOLÓGICA}

Los profesores de idiomas se han hecho conscientes de que una lengua no puede ser aprendida o enseñada sin que se aborde la cultura de la comunidad sujeto de la intervención pedagógica" (Hinkel, 1999, p.2.)

El diseño de esta unidad curricular estuvo orientado bajo los principios del enfoque comunicativo y, por tanto, el método de enseñanza del lenguaje por contenidos orientó la construcción de los materiales empleados en la intervención pedagógica. El método comunicativo de enseñanza del lenguaje (Comunicative Language Teaching) se fundamenta en la teoría del lenguaje como comunicación propuesto por Halliday (1973) en el que el lenguaje es considerado como elemento funcional y por tanto, es aprendido cuando el estudiante lo emplea para comunicarse en situaciones auténticas y significativas de su realidad. Richard \& Rogers (1991) reiteran esta idea al considerar que este enfoque desarrolla también otras habilidades asociadas al lenguaje de tal forma que su aprendizaje se consolida desde una unidad y no como estructuras separadas dando como resultado un verdadero aprendizaje de una lengua. De esta forma, este método permite el planteamiento y desarrollo de actividades contextualizadas relacionadas directamente con el contexto en el cual se desarrolla este estudio. 
La teoría del conocimiento usada en esta unidad curricular es el Constructivismo, desarrollado por Jean Piaget (1952) y Vygotsky (1934), ya que esta teoría también orienta la enseñanza de la institución educativa donde se desarrolló este proyecto. Tal como lo señalan Castillo et all (2017) "para el constructivismo pedagógico (Ausubel, Vygotski o Piaget), la motivación es decisiva a la hora de integrar y consolidar lo que se aprende. El aprendizaje se postula como un producto de una reconstrucción. Cada individuo la va reproduciendo constantemente como resultado de su interacción tanto en los aspectos cognitivos como en los sociales. De esta manera el conocimiento no es una copia fiel de la realidad, sino una reconstrucción del individuo" (p.104).

De esta forma, el conocimiento local se reconoce como resultado de la interacción entre las ideas, conceptos y teorías de los participantes que están siempre presentes durante el proceso de aprendizaje de una nueva lengua. Bajo estas orientaciones teóricas, la unidad curricular presentada en este proyecto se orienta hacia la interacción entre el idioma ingles y el conocimiento local (fósiles, comidas, tradiciones, creencias) desde una estrategia basada en contenidos.

Hablando del diseño de la unidad curricular en mención, éste inició con un análisis de necesidades en el que se determinó la importancia de transformar la instrucción del inglés en una actividad más auténtica y significativa para los estudiantes. Dicho análisis incluyó un estudio de las características de la institución educativa, la población objeto del estudio, el currículo educativo, los planes de área de inglés y las prácticas docentes en el área de inglés. Fruto de este análisis se construye la unidad curricular (ver anexo1) que se desarrollo durante un periodo académico (10 semanas) en el grado noveno.

\section{ESTRATÉGIAS PEDAGÓGICAS-MOMENTOS DE LA EXPERIENCIA}

Esta investigación tiene dos momentos centrales que, a la vez, son los espacios constituidos para el desarrollo de las estrategias pedagógicas. Inicialmente, se emplea el análisis de necesidades desarrollada preliminarmente a la construcción de la unidad curricular como recurso orientador de la construcción temática desde la idea de que "aprender/enseñar implica una participación activa en comunidades sociales, es estar incluido y participar no sólo de los productos culturales, sino también de los procesos sociales de creación de cultura." (Malik Lievano (2010, p.22). En este sentido, el investigador-docente construye la unidad curricular con base en una recopilación documental y testimonial sobre artefactos de la cultura local. En seguida, se hace la implementación pedagógica y, al mismo tiempo, los estudiantes desarrollan un diario de campo en el que registran sus experiencias en la parte pedagógica y de reflexión sobre preguntas orientadoras sobre cultura e identidad cultural. 
En segunda instancia, el investigador-docente, previa lectura de los diarios estudiantiles, y bajo parámetros de regularidad y confiabilidad, determina 5 estudiantes que son objeto del desarrollo de una entrevista general y una focalizada para ahondar en conceptualizaciones desarrolladas en los diarios. De esta forma, con la información obtenida, se crean unas matrices de análisis y se hace la respectiva triangulación de datos para establecer las categorías de análisis que dan cuenta del fenómeno central de este estudio: el posicionamiento cultural e identidad cultural de los participantes.

\section{ANÁLISIS DE DATOS Y RESULTADOS}

En la primera parte de este apartado el lector encuentra una descripción del análisis de datos que permitió el establecimiento de categorías desde la teoría fundamentada como la forma de construir una teoría desde el análisis sistemático de los datos obtenidos (Glaser \& Strauss, 1967). El proceso de análisis y triangulación de datos se explica de la siguiente forma:

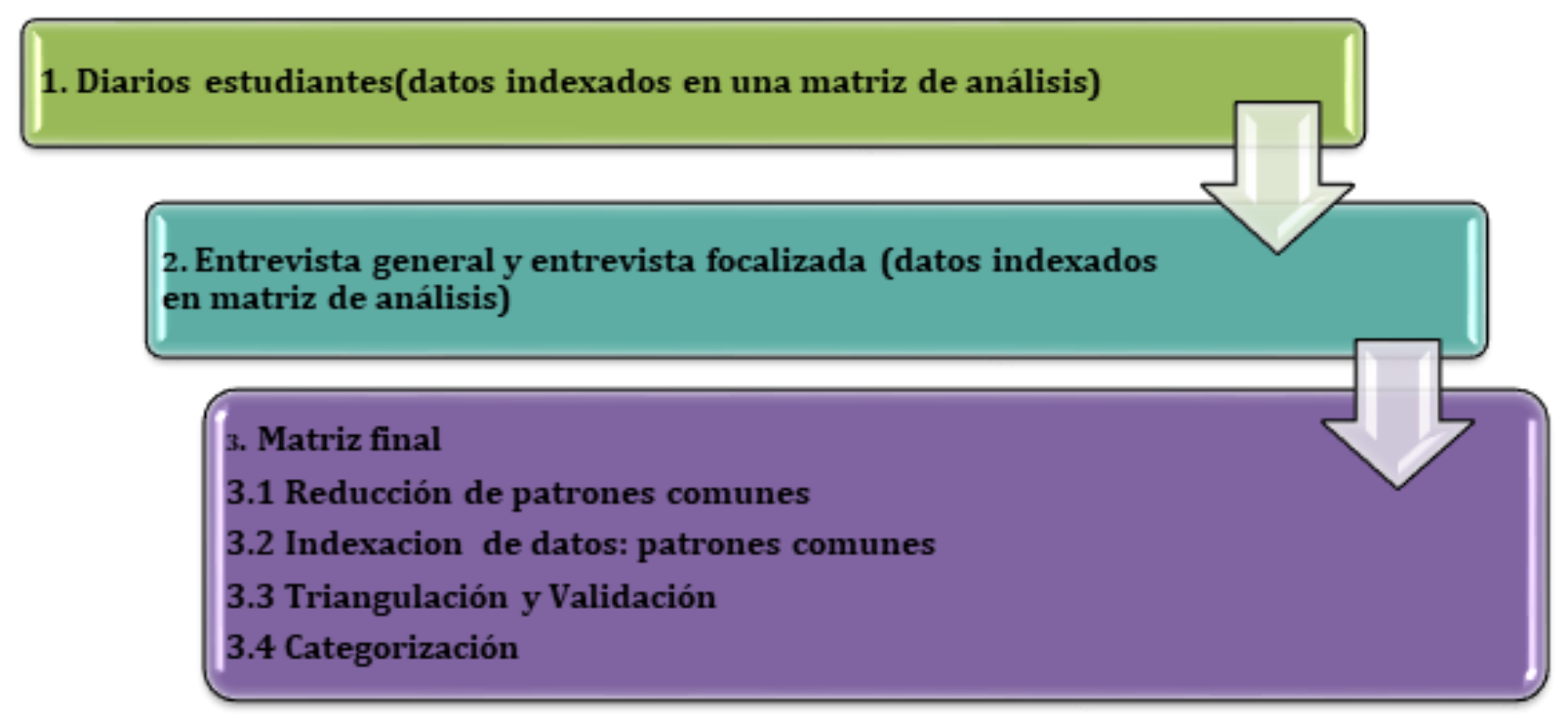

El procedimiento mencionado requirió del investigador-docente mayor sensibilidad y agudeza hacia los conceptos y afirmaciones de los participantes y las relaciones que emergían de los mismos; para ello se siguió un método inductivo donde las categorías emergieron luego de codificar los datos, ejemplo de ello está en las matrices de indexación de datos donde se establecieron patrones de conceptos acompañados de la teoría que explica las categorías y el fenómeno del estudio.

Fruto del análisis explicado, desde la información de los diarios y las entrevistas junto con la teoría fundamentada de las conceptualizaciones, se establecieron dos categorías de análisis que explican el fenómeno de posicionamiento cultural e identidad cultural de los participantes así: 
- Conocer y entender mi cultura: el nombre de esta categoría emerge de las acepciones de los participantes cuando mencionan "Mi Cultura" para mostrar conocimiento y comprensión de los artefactos de su cultura y su posicionamiento como dueños de este historial (Nieto, 2002). Se considera relevante preservar la expresión "mi cultura" ya que representa y da validez a la categoría como representación de las voces de los estudiantes. La naturaleza de esta categoría es la construcción y refuerzo del conocimiento y entendimiento cultural en la vida de los estudiantes para llegar a dar respuesta al interrogante central de la investigación (Cómo el conocimiento cultural es representado por los participantes durante el desarrollo de una unidad curricular basada en contenidos).

Para ser más explícitos frente a esta categoría, reiteramos la idea de que "la cultura no existe en el vacío, sino que está situada en un contexto particular" tal como lo postula Nieto $(2002$, p.11) y de esta forma los participantes usan el contexto de Floresta como delimitante de su conocimiento cultural; este fenómeno requiere tener en cuenta un "sentimiento de pertenecer a" que determina una identidad como miembro de una comunidad y cultura. Los participantes construyen su conocimiento cultural sobre la base de los elementos que provee el contexto y el acercamiento a los mismos. De esta forma los estudiantes muestran como la cultura tiene una fuerte relación con las prácticas y los productos que estructuran un conocimiento cultural cuando afirman:

Js2-12': a Floresta lo identifican los chorotes, la empanada de mora y la sopa de gallina.

Is2: nuestra comida es única por los chorotes, la empanada de mora y el vino que hacen en Cely.

Los participantes del estudio usan saberes específicos para caracterizar aún más sus afirmaciones de los artefactos (comida) como constitutivos de su posicionamiento e identidad cultural; los siguientes ejemplos así lo muestran:

Js6-4: Los chorotes son una comida especial porque solo se preparan una vez al año, en la época en que sale el maíz.

Is6: sus platos típicos representan formas de mi pueblo como el uso de sus productos agrícolas según las cosechas para preparar la comida.

1 Este es el formato usado para indexar los datos en el que "J" significa Diario, "I" representa entrevista. Para tener referencia del participante se emplea la letra "S" acompañada del número asignado a cada uno de ellos y luego del guión el número de la línea en la que se encontró dicha afirmación.

ORATORES ISSN Impreso: 2410-8928 ISSN Electrónico: L-2644-3988 Año 8. Número 12. Junio - Noviembre 2020 
Estas líneas dejen entrever que los participantes también reconocen que su saber está situado y contextualizado. Este conocimiento se consolida desde una práctica heredada que, como lo plantea Riley (2007), se construye sobre la base de los elementos (sociales, políticos, ambientales) disponibles para la comunidad (p.40) cuando aseguran que:

Js3: las arepas eran el único alimento que tenían (sus abuelos) porque en esa época los otros alimentos como el arroz eran muy caros.

Así como los participantes identifican particularidades de su cultura (chorotes, arepas, vino), el conocimiento sobre los artefactos es moldeado por el reconocimiento de las actividades humanas o prácticas diarias como factores asociados a una identidad cultural. Nieto (2002) y Riley (2007) proponen que los factores sociales y económicos determinan el conocimiento cultural de los individuos como se evidencio en los ejemplos anteriores. Este análisis permite afirmar que la cultura está determinada por el uso de las experiencias de vida para establecer un posicionamiento cultural de los participantes.

Este posicionamiento no solo se usa como referentes los artefactos mencionados anteriormente, sino que también incluye otros nuevos; uno de los participantes durante el desarrollo de la unidad curricular declara:

Js4-4: es importante rescatar los fósiles porque son un patrimonio cultural de mi pueblo y porque aqui hay más de 40 grupos de fósiles.

En razón a esta afirmación, la participante amplía su percepción de cultura cuando incluye unos elementos distintos a comidas y tradiciones, en este caso fósiles, como "patrimonio cultural"; este término sugiere que los fósiles tienen un enorme significado para su pueblo y habitantes ya que hacen parte de la historia local. Además, al posicionar los fósiles como patrimonio, el participante también asume una posición crítica al agregar la importancia de cuidar dichos materiales; desde las palabras de Álvarez \& Bonilla (2009), el participante exhibe esta posición no solo basado en juicios personales sino en el análisis y comprensión de su cultura local (p. 153).

Durante el análisis de esta primera categoría, los participantes demostraron "reconocimiento" de los artefactos para establecer un posicionamiento cultural. Heidegren (2004) asevera que este reconocimiento es muy importante en el proceso de socialización y formación de una identidad ya que coopera en la creación de un sentimiento de pertenencia y familiaridad con las prácticas culturales. 
Desde este análisis se estableció la segunda categoría que da cuenta del alcance de la implementación pedagógica

- Mi herencia cultural como componente de mi identidad. Las reflexiones de los participantes en la primera categoría mostraron reconocimiento y comprensión de los elementos culturales de Floresta; ahora, en esta segunda categoría los participantes adoptan posiciones y acciones frente de los artefactos reconocidos como constitutivos de su dimensión cultural. Esta categoría surge del descubrimiento de cómo cada participante adopta la responsabilidad de valorar y preservar los elementos, tradiciones o prácticas que hacen parte de su cultura local así como de los significados atribuidos a cada uno de ellos para establecer una conciencia cultural.

A través del análisis de datos, las voces de los participantes reflejaron una responsabilidad personal asumida para lo que más valoran de su cultura; de esta forma, el nombre de esta categoría da sentido a la posición de preservar una herencia cultural como parte de su identidad. Es, también coherente, con el ideal de Freire, citado por Malik (2013), de una educación liberadora que "pretende desarrollar en el individuo una conciencia crítica que le permita reflexionar sobre una situación, valorarla y valorarse a sí mismo. Las personas, a través de esta conciencia crítica, pueden realizar una verdadera creación cultural, vivir el proceso de su liberación" (p. 23).

Para iniciar con esta categoría, presento al lector los siguientes ejemplos donde se valoran los artefactos culturales dada su naturaleza de construcción social (Nieto, 2002,p.11; Hinkel,1999, p.45):

Js2-2: La música carranguera es una tradición de mi pueblo y habla de ciertas tradiciones nuestras.

Is2: La música que es nuestra tradición es lo que más debemos preservar para que las otras generaciones conozcan nuestra cultura.

Js4-22: Me gusta la letra porque conserva nuestra lengua, la de mi región y mis papás cuando dicen "sumerce, naiden, su persona, ahí mismitico, taluego" y eso es lo que debemos cuidar.

Is4: la música es nuestra riqueza porque ésta habla sobre los paisajes cómo es la gente, qué suelen hacer y cuáles son sus trabajos diarios. Así como en la canción del caballito de acero que trabajamos en clase.

En estos ejemplos, los participantes reconocen el valor de la música para transmitir la cultura a las futuras generaciones y, de esta forma, establecen una relación entre el producto (música) y

ORATORES ISSN Impreso: 2410-8928 ISSN Electrónico: L-2644-3988 Año 8. Número 12. Junio - Noviembre 2020 
las prácticas (uso de la música para transmitir costumbres) lo cual explica que los estudiantes son conscientes de la importancia de dicha relación para preservar su cultural y reiteran la teoría de la cultura como construcción social que da cuenta de la historia y valores alrededor de una herencia cultural particular (Riley, 2007).

También se hace evidente, en el caso del estudiante $n^{\circ} 4$, que el lenguaje se usa preservar tradiciones y, más trascendente aún, para mantener una tradición lingüística de su contexto como marca de su dimensión cultural. Riley (2007) argumenta que el principal mecanismo de conocimiento social es el lenguaje ya que éste codifica y preserva la cultura como saber y provee un medio para ser transmitida y definida continuamente (p.53). Examinando otros ejemplos, los estudiantes consideran el conocimiento antiguo o tradicional como algo muy importante de preservar:

Js1: La gente antigua enseña a sus hijos a preparar los platos típicos porque es típico de nuestra región, es una tradición para mantener.

En el análisis de esta categoría, las reflexiones de los participantes revelan que ellos se vuelven conscientes de su dimensión cultural cada vez que asumen una posición de dueños, no solo de los artefactos, sino de las prácticas sociales de su cultura. Adicional, estas reflexiones muestran como las instituciones (familia y municipalidad) y las prácticas (compartidas o transmitidas) ayudan a los participantes a dar cuenta de su propia percepción de cultura.

Basados en lo declarado en la fundamentación teórica, la identidad no es una simple entidad ya que está formada por diversos factores (sociales, religiosos, ideológicos, políticos, culturales entre otros); por esta razón los participantes se refieren a una construcción de identidad al tiempo que reconocen la dimensión social de la cultura como ejes de desarrollo de una identidad cultural propia.

\section{CONCLUSIONES}

El proceso de entender la cultura de los participantes requiere un entendimiento de la cultura como elemento heredado pero que también es negociado y está permeado por diversas instituciones y experiencias a lo largo de la vida.

Durante el desarrollo de la intervención pedagógica se mantuvo presente la importancia de abordar artefactos culturales representativos del contexto para proponer las actividades de aprendizaje del inglés y que, igualmente, permitieran generan reflexión de los estudiantes sobre su posicionamiento e identidad cultural. Tal como lo plantea Forés "Una de las funciones de 
nuestra profesión docente es acompañar en la construcción de la identidad del sujeto individual y colectivo" (p. 114) y ese fue el eje central de la función pedagógica de dicha intervención. A través de la implementación de una unidad curricular basada en contenidos (cultura florestana) los participantes dieron cuenta de las representaciones culturales propias de su región y de un avance significativo de desempeño en el uso del inglés como lengua extranjera.

El grado de posicionamiento de los estudiantes determinó la manera en que ellos dieron cuenta de su herencia cultural; en todos los fragmentos se evidenció un grado de sentimiento de afiliación y reconocimiento de prácticas sociales, artefactos, tradiciones familiares, usos lingüísticos y sus propias experiencias como elementos de una cultura propia. De la misma manera, los participantes mostraron una comprensión de su rol como portadores de una historia cultural que desean preservar en sus vidas.

En el desarrollo de este estudio también se encontró que los participantes relacionan su conocimiento cultural con una dimensión social que da testimonio de tradición cultural con antecedentes sociales, históricos, ambientales y familiares.

Al hablar de las conclusiones pedagógicas de esta intervención es fundamental reconocer que aproximarse a una cultura a través de la enseñanza de una segunda lengua requiere conocimiento no solo del campo pedagógico-didáctico sino también del campo sociolingüístico y sociocultural; esto debido a que las prácticas pedagógicas se constituyen en medios con los cuales el investigadordocente de aproxima a la comprensión de un fenómeno social.

Ya que este estudio no reporta resultados de la intervención pedagógica en el área de inglés como objetivo central, los resultados muestran la importancia de involucrar la realidad de los estudiantes en las prácticas curriculares para crear espacios donde los estudiantes sean conscientes, reflexionen y den sentido a sus propias vidas desde el desarrollo de una temática de clase.

Basados en los resultados de este estudio, donde los estudiantes usaron exitosamente el lenguaje para crear conciencia de su dimensión cultural, el investigador-docente considera pertinente abogar por la implementación de prácticas pedagógicas donde el contexto local sea el mediador para alcanzar objetivos lingüísticos; en la implementación pedagógica de este estudio se mostró que el aprendizaje de un idioma no se puede dar fuera de un contexto de aprendizaje y que abordar la cultura local permitió un incremento en la motivación y compromiso de los participantes dada la familiaridad y relevancia de los temas abordados en clase. 
Este estudio contribuyó, también, al campo de la investigación, particularmente el de la sociolingüística, ya que abordó el uso del lenguaje para la comprensión de un fenómeno social y cultural demostrando cómo mediante el lenguaje los individuos pueden ser representados como miembros de un grupo social especifico. El uso de la investigación cualitativa y el estudio de caso para estudiar el fenómeno en su escenario natural permitieron construir una comprensión de la cultura de los participantes mediante el análisis de la información; adicionalmente, la teoría fundamentada se construyó a la par del desarrollo del estudio en aras de obtener comprensión del fenómeno de estudio.

\section{REFERENCIAS BIBLIOGRÁFICAS}

Alvarez, J \& Bonilla, X. (2009). Adressing culture in the EFL classroom: A Dialogic Proposal. In Profile Vol. 11, $\mathrm{N}^{\mathrm{o}} 2$.

Byram, M., \& Risager, A. (1999). Language teachers, politics and cultures. Clevelon: Multilingual Matters Ltd.

Burns, R (2000) Introduction to Research Methods, London, Sage.

Denzin, Norman K. \& Lincoln, Yvonna S. (2005). The Sage Handbook of Qualitative Research. Third Edition. Thousand Oaks: Sage Publications, Inc. Introduction. The Discipline and Practice of Qualitative Research: pp. 1-13.

Hinkel, Eli. (1999). Culture in the Second Language Teaching and Learning. Cambridge University Press.

Hodge, Robert and Kress, Gunther. (1999). Language as Ideology. Routledge Taylor \& Francis Group. (p.1)

Moran, P. (2001). Teaching Culture: Perspectives in Practice. Heinle \& Heinle. New York.

Nieto, Sonia. (2002) Language, Culture and Teaching, Critical Perspectives for a new century. Lawrence Erlbaum Associates Publishers. Mahwah, New Yersey.

Riley, Philip. ((2007). Language, Culture and Identity. Athenaeum Press Ltd, Gateshead Tyne \& Wear. 
Yin, Robert. Application of case study research.(2012). Third edition. Thousand Oaks: Sage Publications, Inc.

Zuluaga, Carmen, López, Margarita \& others. (2009)Integrating the coffee culture with the Teaching of English. In Profile vol ${ }^{\circ} 11 \mathrm{n}^{\circ} 2$.

\section{WEBGRAFÍA}

Castillo Carbonel, Miguel, Paredes Navarro, Lydia and Bou Blanco, Mireia. Escuela y educacion social: necesidades, contextos y experiencias. Barcelona, ESPAÑA: Editorial UOC, 2017. ProQuest ebrary, Web. 5 July 2017. Copyright 2017. EditorialUOC. All rights reserved.

Forés, Anna, and Novella, Ana María, eds. 7 retos para la educación social: reinventarse como profesional de lo social, nuevos desafíos para la empleabilidad. Barcelona, ESPAÑA: Editorial Gedisa, 2013. ProQuest ebrary. Web. 17 July 2017.

Copyright (C) 2013. Editorial Gedisa. All rights reserved.

Harwell, S. (1997). Project-based learning. In W.E. Blank \& S. Harwell (Eds.), Promising practices for connecting high school to the real world (pp. 23-28). Tampa, FL: University of South Florida. (ERIC Document Reproduction Service No. ED407586).

Klug, Ron (2002) How to Keep a Spiritual Journal. A guide to journal keeping for inner growth and personal discovery from http://books.google.com.co/books

Malik Liévano, Beatriz, Senra Varela, Maria, and Mamolar Alarcón, Pilar. Asesoramiento y consulta en educación social. Madrid, ES: UNED - Universidad Nacional de Educación a Distancia, 2013. ProQuest ebrary. Web. 10 July 2017.Copyright (C 2013. UNED - Universidad Nacional de Educación a Distancia. All rights reserved.

Norton, Bonny. (1997). Language, Identity, and the Ownership of English. TESOL Quarterly , Vol. 31, No. 3, Language and Identity (Autumn, 1997), pp. 409-429. Publicado por: Teachers of English to Speakers of Other Languages, Inc. (TESOL) Artiulo disponible en URL: http://www. jstor.org/stable/3587831

Norton, B., \& Kamal, F. (2003). The imagined communities of English language learners in a Pakistani school. Journal of Language, Identity, and Education, 2(4), 301-307. Articulo disponible en URL: : http://educ.ubc.ca/faculty/norton/ 